\title{
Determination of all stability region of unstable fractional order system in case of bearing system
}

\author{
Phu Tran Tin ${ }^{1}$, Nguyen Quang Dung ${ }^{2}$, Minh Tran ${ }^{3}$, Van-Duc Phan ${ }^{4}$, Hoang-Nam Nguyen ${ }^{5}$, Tran \\ Thanh Trang 6 \\ ${ }^{1}$ Wireless Communications Research Group, Faculty of Electrical and Electronics Engineering, \\ Ton Duc Thang University, Ho Chi Minh City, Vietnam \\ ${ }^{2}$ Faculty of Electrical and Electronics Engineering, Ton Duc Thang University, Ho Chi Minh City, Vietnam \\ ${ }^{3}$ Optoelectronics Research Group, Faculty of Electrical and Electronics Engineering, Ton Duc Thang University, \\ Ho Chi Minh City, Vietnam \\ ${ }^{4}$ Center of Excellence for Automation and Precision Mechanical Engineering, Nguyen Tat Thanh University, \\ Ho Chi Minh City, Vietnam \\ ${ }^{5}$ Modeling Evolutionary Algorithms Simulation and Artificial Intelligence, Faculty of Electrical \&amp; Electronics \\ Engineering, Ton Duc Thang University, Ho Chi Minh City, Vietnam \\ ${ }^{6}$ Faculty of Electrical and Electronics Engineering, Ho Chi Minh City University of Food Industry, 140 Le Trong Tan, \\ Ho Chi Minh City, Vietnam.
}

\begin{tabular}{l}
\hline Article Info \\
\hline Article history: \\
Received Dec 18, 2018 \\
Revised Mar 1, 2019 \\
Accepted May 28, 2019 \\
\hline Keywords: \\
Control system \\
PID \\
RIM \\
Unstable fractional order \\
system
\end{tabular}

\begin{abstract}
In this paper, we propose and investigate the optimal tuning PID controller of unstable fractional order system by desired transient characteristics using the real interpolation method (RIM). The research shows that the main advantages of this method are drawn as the followings: 1) Carrying out an investigation of the stable region of coefficients of a PID controller using D-decomposition method; 2) Applying the method to investigate an unstable fractional order system.
\end{abstract}

Corresponding Author:

Van-Duc Phan

Center of Excellence for Automation and Precision Mechanical Engineering,

Nguyen Tat Thanh University, Ho Chi Minh City, Vietnam

Email:pvduc@ntt.edu.vn

\section{INTRODUCTION}

Due to the individual merits such as no contact, no wear, no lubrication and adjustable dynamics, magnetic bearings (MBs) are being widely applied to a variety of industrial products, especially the highspeed rotating machinery and precise positioning systems [1-3]. Considering the inherent nonlinearity and open-loop instability of MBs, the accurate modeling and control are two essential aspects of achieving highperformance requirements. Nowadays, PID controllers have received considerable attention in the last years both from an academic and industrial point of view [1-5]. In fact, in principle, they provide more flexibility in the controller design, concerning the standard PID controllers, because they have five parameters to select. However, this also implies that the tuning of the controller can be much more complicated. They have been successfully applied in practical applications such as motion control of manipulators and chaos control of electrical circuits. In these applications, it has been verified that PID controllers can improve the performance of traditional control system adopting integer order PID controllers. The most important advantage of the PID controllers is that they can afford broader possibilities offered by their new fractional order dynamics [4-7].

Journal homepage: http://iaescore.com/journals/index.php/IJPEDS 
However, this also indicates that the tuning strategies of PID controllers are much more complicated. In the researches on the PID controllers, tuning of controller parameters has become a significant issue.

In general, the tuning methods for PID controllers are classified into analytical, numerical, and rulebased ones. In [6-7] the controller parameters have been analytically derived by solving nonlinear equations fulfilling the gain/phase crossover frequency and phase/gain margin specifications. The robustness to loop gain variations specification proposed in [8] has also been widely used to design PID and proportionalintegral (PI) controllers. The merits of the analytical method are apparent; however, it is available only when the equations are few in number and simple. Therefore, it is challenging to obtain a complete PID controller for the MB system by solving five complicated nonlinear equations. As for the rule-based method, it can easily calculate the controller parameters based on empirical tuning rules, which can be observed in [9-21]. In this paper, we propose and investigate the optimal tuning PID controller of unstable fractional order system by desired transient characteristics using the real interpolation method (RIM). The main advantages of this method are drawn as the followings:

1) Carrying out an investigation of the stable region of coefficients of a PID controller using Ddecomposition method.

2) Applying the method to investigate an unstable fractional order system.

The rest of this paper is organized as follows. In Section 2 the problem is formulated. Numerical results and discussions are described in Section 3. Conclusions are drawn in Section 4.

\section{PROBLEM FORMULATION}

In this section, Figure 1 illustrates the control system with the negative unity feedback. The mathematical description of this model is presented.

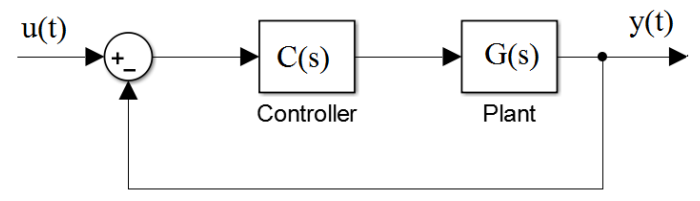

Figure 1. Control system with negative unity feedback

The transfer function of the PID controller can be formulated as

$$
C(\mathrm{~s})=\mathrm{K}_{p}+\frac{\mathrm{K}_{i}}{s}+\mathrm{K}_{d} s
$$

The transfer function of the plant (in the fractional form) is calculated as

$$
\mathrm{G}(\mathrm{s})=\frac{\mathrm{B}_{G}(\mathrm{~s})}{A_{G}(s)}=\frac{b_{n} s^{\beta_{n}}+b_{n-1} s^{\beta_{n-1}}+\cdots+b_{1} s^{\beta_{1}}+b_{0} s^{\beta_{0}}}{a_{m} s^{\alpha_{m}}+a_{m-1} s^{\alpha_{m-1}}+\cdots+a_{1} s^{\alpha_{1}}+a_{0} s^{\alpha_{0}}}
$$

where $m, n \in N$, and $a_{0}, \ldots, a_{m}, b_{0}, \ldots, b_{n}, \alpha_{m}>\alpha_{m-1}>\cdots>\alpha_{1}>\alpha_{0}=0, \beta_{n}>\beta_{n-1}>\cdots>\beta_{1}>\beta_{0}=$ 0 are arbitrary real numbers $a_{m} \neq 0, b_{n} \neq 0$.

The transfer function of PID controller (1) can be rewritten in the rational form as the following

$$
C(\mathrm{~s})=\frac{B_{C}(s)}{A_{C}(s)}=\frac{\mathrm{K}_{d} s^{2}+\mathrm{K}_{p} s+\mathrm{K}_{i}}{s}
$$

The characteristic equation in this analysis can be expressed as

$$
\mathrm{W}(s)=1+C(\mathrm{~s}) \mathrm{G}(\mathrm{s})
$$

Characteristic polynomial is formulated as 


$$
\mathrm{P}(s)=s A_{G}(s)+\left(\mathrm{K}_{d} s^{2}+\mathrm{K}_{p} s+\mathrm{K}_{i}\right) \mathrm{B}_{G}(s)
$$

A gain-phase margin tester (GPMT) can be thought of as a "virtual compensator", provides information for plotting the boundaries of constant gain margin and phase margin in a parameter plane [14]. The frequency independent GPMT is given as in [15]:

$$
G_{t}(\mathrm{M}, \phi)=\mathrm{Me}^{-j \phi}
$$

For a given IOPID controller parameters $\mathrm{K}_{p}, \mathrm{~K}_{i}, \mathrm{~K}_{d}$ the closed-loop system is said to be boundedinput bounded-output (BIBO) stable if the quasi-polynomial $\mathrm{P}\left(\mathrm{s}, \mathrm{K}_{p}, \mathrm{~K}_{i}, \mathrm{~K}_{d}\right)$ has no roots in the closed righthalf of the s-plane (RHP). The stability domain $\mathbf{S}$ in the parameter space $\mathrm{P}$ with $\mathrm{K}_{p}, \mathrm{~K}_{i}, \mathrm{~K}_{d}$ being coordinates is the region that for $\mathrm{K}_{p}, \mathrm{~K}_{i}, \mathrm{~K}_{d} \in S$ the roots of quasi-polynomial $\mathrm{P}\left(\mathrm{s}, \mathrm{K}_{p}, \mathrm{~K}_{i}, \mathrm{~K}_{d}\right)$ all lie in open left-half of the s-plane (LHP). The boundaries of the stability domain $\mathrm{S}$ which are described by real root boundary (RRB), infinite root boundary (IRB) and complex root boundary (CRB) can be determined by the D-decomposition method [15], [16] . These boundaries are defined by the equations $\mathrm{P}(0, \mathrm{~K})=0, \mathrm{P}(\infty, \mathrm{K})=0$ and $P( \pm j \omega, K)=0$ for $\omega \in(0, \infty)$, respectively, where $P(s, K)$ is the characteristic function of the closedloop system and $\mathrm{K}$ the vector of controller parameters.

\subsection{Determining RRB}

In applying the descriptions of stability boundaries of the stability domain S to the FOCE in (1), the RRB turns out to be simply a straight line given by

$$
\mathrm{P}\left(0, \mathrm{~K}_{p}, \mathrm{~K}_{i}, \mathrm{~K}_{d}\right)=0 \Rightarrow K_{i}=0
$$

for $s^{\beta_{0}}=1$ in the transfer function of the plant in (2).

\subsection{Determining IRB}

The calculating of the IRB faces with more technical difficulties due to the fractional component. FOCE possesses an infinite number of roots, which cannot be calculated analytically in the general case. However, the asymptotic location of roots far from the origin is well known [17], which may lead to IRB. The objective of this section is to determine the stabilizing region in $\left(K_{p}, K_{i}\right)$ plane with given $K_{d}$, and values for which the following complex polynomial is Hurwitz:

$$
\mathrm{D}(s)=s L(s) A_{G}(s)+\left(\mathrm{K}_{d} s^{2}+\mathrm{K}_{p} s+\mathrm{K}_{i}\right) \mathrm{M}(\mathrm{s}) \mathrm{B}_{G}(s)
$$

where $\mathrm{L}(\mathrm{s})$ and $\mathrm{M}(\mathrm{s})$ are given complex fractional order polynomials. When $L(s)=M(s)=1$, the stabilization of Eq. (8) reduces to the standard IOPID stabilization.

From (8), we have $\mathrm{D}(\infty)=0$. Suppose when $s \rightarrow \infty,\left[L(s) A_{G}(s)\right] /\left[M(s) B_{G}(s)\right] \rightarrow c s^{t}$ where $\mathrm{t}$ and $\mathrm{c}$ are real and complex numbers respectively. We have [16]:

(a) If $t \neq 1$, then the boundary does not exist.

(b) If $t=1$ and $\mathrm{c}$ is not real, then the boundary does not exist.

(c) If $t=1$ and $\mathrm{c}$ is real, then

$$
\begin{aligned}
& \lim _{s \rightarrow \infty} \frac{D(s)}{s L(s) A_{G}(s)}=\lim _{s \rightarrow \infty} 1+\frac{N(s)\left(K_{p} s+K_{i}+K_{d} s^{2}\right)}{s A_{G}(s)}=1+K_{d} c=0 \\
& K_{d}=-\frac{1}{c}
\end{aligned}
$$

The RRB and IRB lines can be obtained from (7) and (10), respectively.

\subsection{Determining CRB}

Int J Pow Elec \& Dri Syst Vol. 10, No. 4, Dec 2019 : 1734 - 1741 
To construct the CRB, we substitute $s=j \omega$ into (5) to obtain

$\mathrm{P}(\mathrm{j} \omega)=A_{C}(\mathrm{j} \omega) A_{G}(\mathrm{j} \omega)+B_{C}(\mathrm{j} \omega) \mathrm{B}_{G}(\mathrm{j} \omega)=0$

Using D-decomposition to find stability [15]. In the s plane $j^{\varphi}=e^{j\left(\frac{\pi}{2}\right) \varphi}=\cos \left(\frac{\pi}{2} \varphi\right)+\mathrm{j} \sin \left(\frac{\pi}{2} \varphi\right)$, where $\varphi$ is a real number. The formula (6) can be expressed into a formula with the separate real and imaginary components

$$
\mathrm{P}(\mathrm{j} \omega)=\left[\left(\mathrm{K}_{i}-\mathrm{K}_{d} \omega^{2}\right) \mathrm{R}_{B G}(\omega)-\omega \mathrm{I}_{A G}(\omega)\right]+j \omega\left[\mathrm{K}_{p} \mathrm{R}_{B G}(\omega)+\mathrm{R}_{A G}(\omega)\right]=0,
$$

where $\mathrm{R}_{B G}, \mathrm{R}_{A G}, \mathrm{I}_{A G}$ are the real component of $\mathrm{B}_{G}(\mathrm{j} \omega)$, the real and imaginary components of $A_{G}(\mathrm{j} \omega)$, respectively. detail form:

Finally, by setting the real and imaginary parts equalized zero the formula (12) leads to the

$$
\left\{\begin{array}{l}
\mathrm{R}_{A G}(\omega)+\mathrm{K}_{p} \mathrm{R}_{B G}(\omega)=0 \\
\left(\mathrm{~K}_{i}-\mathrm{K}_{d} \omega^{2}\right) \mathrm{R}_{B G}(\omega)-\omega \mathrm{I}_{A G}(\omega)=0
\end{array}\right.
$$

\section{The solution in the $\left(K_{p}, K_{i}\right)$ plane}

This is a three-dimensional system in terms of the controller parameters $\mathrm{K}_{p}, \mathrm{~K}_{i}, \mathrm{~K}_{d}$. In order to deal with two unknowns $\mathrm{K}_{p}, \mathrm{~K}_{i}$, the value of $\mathrm{K}_{d}$ will be fixed to find the stability region in the $\left(\mathrm{K}_{p}, \mathrm{~K}_{i}\right)$ plane.

Equation (11) rewrite in terms of two unknowns $\mathrm{K}_{p}, \mathrm{~K}_{i}$.

$$
\left\{\begin{array}{l}
\mathrm{K}_{p}=-\mathrm{R}_{A G}(\omega) / \mathrm{R}_{B G}(\omega) \\
\mathrm{K}_{i}=\omega I_{A G}(\omega) / \mathrm{R}_{B G}(\omega)+\mathrm{K}_{d} \omega^{2}
\end{array}\right.
$$

The above two equations trace out a curve in the $\left(\mathrm{K}_{p}, \mathrm{~K}_{i}\right)$-plane representing the CRB, for fixed $\mathrm{K}_{d}$, as $\omega$ run from 0 to $\infty$.

\section{The solution in the $\left(K_{p}, K_{d}\right)$ plane}

Next, to find the stability region in the $\left(K_{p}, K_{d}\right)$ plane, the value of $K_{i}$ will be fixed. Then formula (13) can be rewritten as:

$$
\left\{\begin{array}{l}
\mathrm{K}_{p}=-\mathrm{R}_{A G}(\omega) / \mathrm{R}_{B G}(\omega) \\
\mathrm{K}_{d}=\left[\mathrm{K}_{i}-\omega \mathrm{I}_{A G}(\omega) / \mathrm{R}_{B G}(\omega)\right] / \omega^{2}
\end{array}\right.
$$

The above two equations trace out a curve in the $\left(\mathrm{K}_{p}, \mathrm{~K}_{d}\right)$-plane representing the CRB, for fixed $\mathrm{K}_{i}$, as $\omega$ run from 0 to $\infty$

The solution in the $\left(K_{i}, K_{d}\right)$ plane

Next, to find the stability region in the $\left(K_{i}, K_{d}\right)$ plane, the value of $K_{d}$ will be fixed. Then formula (13) can be rewritten as:

$$
\left\{\begin{array}{l}
\mathrm{K}_{p}=-\mathrm{R}_{A G}(\omega) / \mathrm{R}_{B G}(\omega) \\
\mathrm{K}_{d}=\left[\mathrm{K}_{i}-\omega \mathrm{I}_{A G}(\omega) / \mathrm{R}_{B G}(\omega)\right] / \omega^{2}
\end{array}\right.
$$

The above two equations trace out a curve in the $\left(\mathrm{K}_{i}, \mathrm{~K}_{d}\right)$-plane representing the $\mathrm{CRB}$, for fixed $\mathrm{K}_{p}$, as $\omega$ run from 0 to $\infty$.

\section{NUMERICAL SAMPLE AND DISCUSSION}

Given fractional order transfer function of an unstable bearing system [18] [19], 


$$
\mathrm{G}(\mathrm{s})=\frac{6438}{s^{2.76}+330.04 s^{1.81}-84268.85 s^{0.82}-15869154.14}
$$

Investigation of the stability region of the unstable fractional order transfer function of the bearing system (17) is carried out in the following parts.

\subsection{Determining RRB}

It is important to determine the stability region of the PID controller, tuned for the system (17). The RRB can be determined by (7) as the bellow

$$
K_{i}=0
$$

\subsection{Determining IRB}

The IRB can be determined according to (9)

$$
\left.\lim _{s \rightarrow \infty} \frac{\delta(s)}{s D(s)}=\lim _{s \rightarrow \infty} 1+\frac{643.8\left(K_{p} s+K_{i}+K_{d} s^{2}\right)}{s\left(s^{2.76}+330.04 s^{1.81}-84268.85 s^{0.82}-15869154.14\right)}\right)=1
$$

Not exist the IRB.

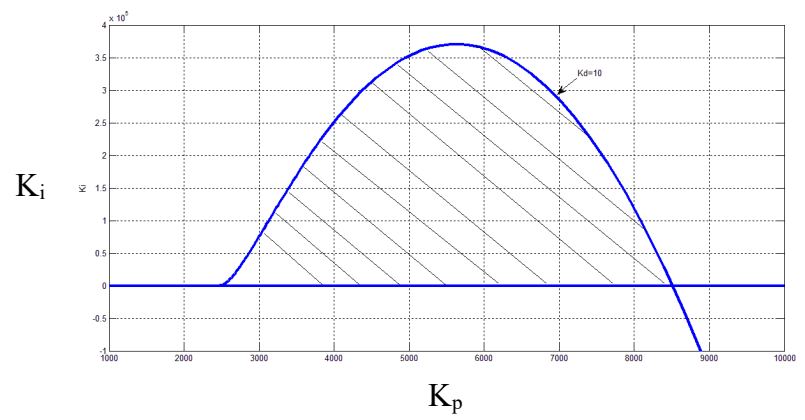

Figure 2. The CRB graph in the $\left(\mathrm{K}_{\mathrm{p}}, \mathrm{K}_{\mathrm{i}}\right)$ plane and $\mathrm{K}_{\mathrm{d}}=10$.

\subsection{Determining CRB}

The CRB can be determined by three conditions (13), (14) and (15). Figure 2 shows the stability region of the transfer function (17). The $\mathrm{CRB}$ in the $\left(\mathrm{K} \_, \mathrm{K} \_\mathrm{i}\right)$ plane when $\mathrm{K}_{-} \mathrm{d}=10$ is shown in the Figure 2. The cross-line area is a feasible region for the coefficients of the PID controller. The cross-line region demonstrates stability region of $\mathrm{K} \_\mathrm{p}, \mathrm{K} \_\mathrm{i}$ where $\mathrm{K} \_\mathrm{d}=10$. Obviously, the stability region of PID controller's coefficients lie in the first quarter of the coordinate [22-25].

Furthermore, Figure 3 shows a more feasible area with five values of $K_{d}$.

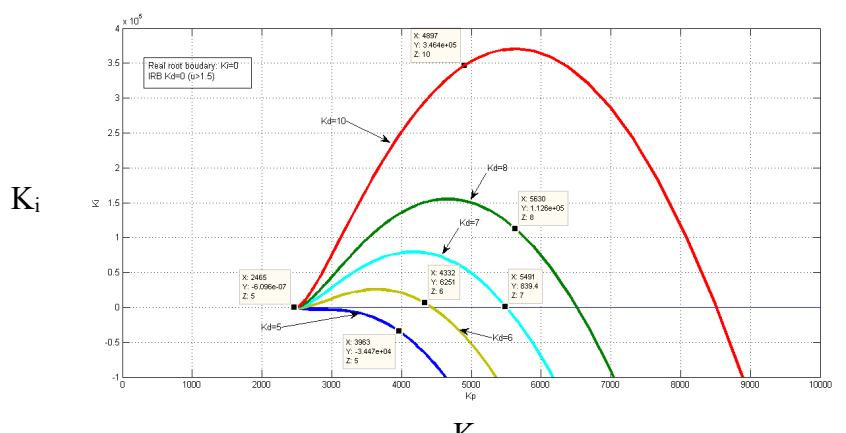

$\mathrm{K}_{\mathrm{p}}$ 
Figure 3. The CRB graphs in the $\left(\mathrm{K}_{\mathrm{p}}, \mathrm{K}_{\mathrm{i}}\right)$ plane with 5 values of coefficients $\mathrm{K}_{\mathrm{d}}$.

Figure 3 shows that by increasing the value of the coefficient $K_{d}$, the feasible region is expanded.

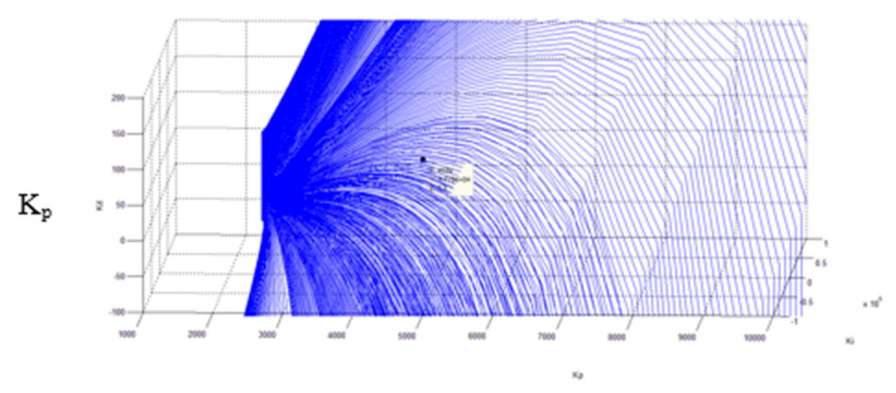

Figure 4. The CRB of the PID coefficients

In Figure 4. The distribution of $K_{p}, K_{i}, K_{d}$ in the 3-D system is represented. In more details, we can investigate the stability region of the PID controller's coefficient separately in the 2-D coordinate plane. In the Figure 4.

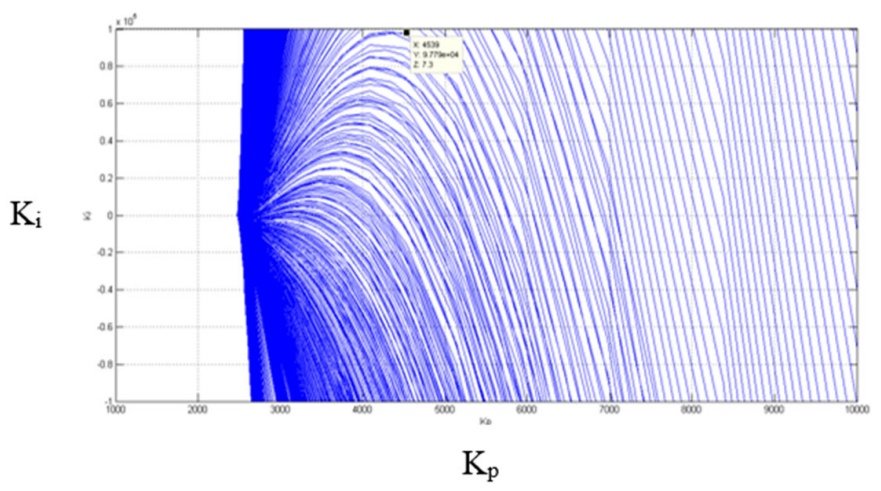

Figure 5. The CRB in the $\left(\mathrm{K}_{\mathrm{p}}, \mathrm{K}_{\mathrm{i}}\right)$ plane

Figure 5 illustrates the stability region of $K_{p}$ and $K_{i}$. Obviously, the stability region is covered the first and fourth quarters of the coordinate system. From the Figure 5, there is a significance that the coefficient $K_{p}>=2465$. Furthermore, The Figure 6 show the stability region in the $K_{p}, K_{d}$ plane. The values of the coefficient $K_{p}, K_{d}$ are located in the first and fourth quarters, and of course $K_{p} \geq 2456$.

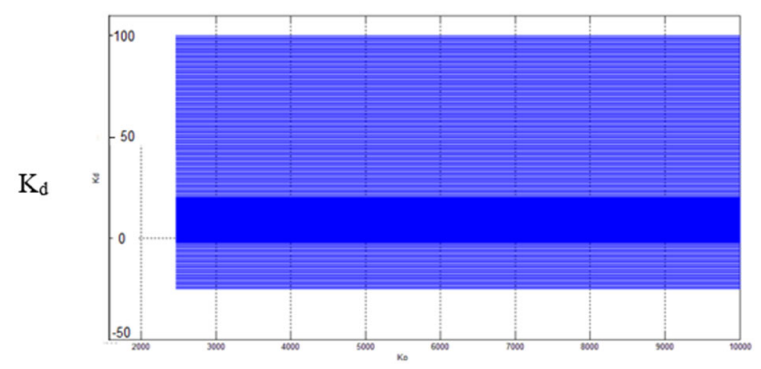

$\mathrm{K}_{\mathrm{p}}$

Figure 6. The CRB in the $\left(\mathrm{K}_{\mathrm{p}}, \mathrm{K}_{\mathrm{d}}\right)$ plane 
The CRB in the $\left(\mathrm{K}_{\mathrm{i}}, \mathrm{K}_{\mathrm{d}}\right)$ plane shows that the distribution of $K_{i}$ and $K_{d}$

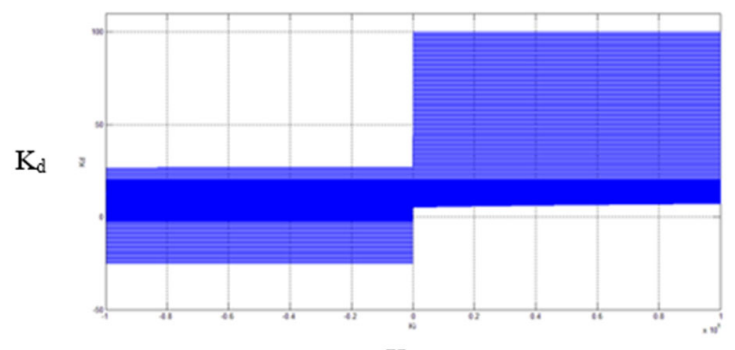

$\mathrm{K}_{\mathrm{i}}$

Figure 7. The CRB in the $\left(\mathrm{K}_{\mathrm{i}}, \mathrm{K}_{\mathrm{d}}\right)$ plane

The results simply lead to the conclusion

$$
\left\{\begin{array}{l}
K_{p}>=2465 \\
K_{i}>0
\end{array}\right.
$$

Three parameters $K_{p}, K_{i}, K_{d}$ of the PID controller are determined in four figure 4-7 [22-25].

\section{CONCLUSION}

In this paper, we propose and investigate optimal tuning PID controller of unstable fractional order system by desired transient characteristics using the real interpolation method (RIM). The research shows that the main advantages of this method are drawn as the followings: 1) Carrying out an investigation of the stable region of coefficients of a PID controller using D-decomposition method; 2) Applying the method to investigate an unstable fractional order system.

\section{ACKNOWLEDGEMENTS}

This research was supported by National Key Laboratory of Digital Control and System Engineering (DCSELAB), HCMUT, VNU-HCM, Vietnam.

\section{REFERENCES}

[1] Heydarpoor, Saeed, and Mohammad Tabatabaei, "Velocity control of a DC motor based on fractional order PI and IMC-based fractional order controllers," International Journal of Advanced Mechatronic Systems, vol. 7, no. 1, 2016. doi:10.1504/ijamechs.2016.079650.

[2] Zheng, Shiqi, "Robust stability of fractional order system with general interval uncertainties," Systems \&amp; Control Letters, vol. 99, pp. 1-8, 2017. doi:10.1016/j.sysconle.2016.11.001.

[3] Vinagre, Blas M., et al, "Fractional PID controllers for industry application. A brief introduction," Journal of Vibration and Control, vol. 13, no. 9-10, pp. 1419-1429, 2007. doi:10.1177/1077546307077498.

[4] Monje, Concepción A., et al., "Tuning and auto-tuning of fractional order controllers for industry applications," Control Engineering Practice, vol. 16, no. 7, pp. 798-812, 2008. doi:10.1016/j.conengprac.2007.08.006.

[5] Chen, Yangquan, et al., "Practical tuning rule development for fractional order proportional and integral controllers," Journal of Computational and Nonlinear Dynamics, vol. 3, no. 2, 2008. doi:10.1115/1.2833934.

[6] Petráš, Ivo, "Tuning and implementation methods for fractional-order controllers," Fractional Calculus and Applied Analysis, vol. 15, no. 2, 2012. doi:10.2478/s13540-012-0021-4.

[7] Li, Hongsheng, Ying Luo, and Yangquan Chen, "A fractional order proportional and derivative (FOPD) motion controller: Tuning rule and experiments," IEEE Transactions on Control Systems Technology, vol. 18, no. 2, 51620, 2010. doi:10.1109/tcst.2009.2019120.

[8] Luo, Ying, Chunyang Wang, and Yangquan Chen, "Tuning fractional order proportional integral controllers for fractional order systems," 2009 Chinese Control and Decision Conference, vol. 6, 2009. doi:10.1109/ccdc.2009.5195101. 
[9] Malek, Hadi, Ying Luo, and Yangquan Chen, "Identification and tuning fractional order proportional integral controllers for time delayed systems with a fractional pole," Mechatronics, vol. 23, no. 7, pp. 746-54, 2013. doi:10.1016/j.mechatronics.2013.02.005.

[10] Valério, Duarte, and José Sá Da Costa, "Tuning of fractional PID controllers with Ziegler-Nichols-type rules," Signal Processing, vol. 86, no. 10, pp. 2771-784, 2006. doi:10.1016/j.sigpro.2006.02.020.

[11] Bhambhani, Varsha, Yangquan Chen, and Dingyü Xue, "Optimal fractional order proportional integral controller for varying time-delay systems," IFAC Proceedings, vol. 41, no. 2, 4910-915, 2008. doi:10.3182/20080706-5-kr1001.00825 .

[12] Chen, Yangquan, Tripti Bhaskaran, and Dingyü Xue, "Practical tuning rule development for fractional order proportional and integral controllers," Journal of Computational and Nonlinear Dynamics, vol. 3, no. 2, 2008. doi:10.1115/1.2833934.

[13] Goncharov, Nguyen Quang Dung, Than Viet Dung, Identification of object with distributed parameters, Theory and practice in the physical, mathematical and technical sciences-XXIV International Scientific and Practice Conference, London, May, 2012. doi:10.18007/gisap:tsca.v0i8.1421.

[14] C.-H. Chang and K.-W. Han, "Gain margins and phase margins for control systems with adjustable parameters," $J$. Guid., Control, Dyn., vol. 13, no. 3, pp. 404-408, 1990.

[15] Hamamci, S.e., "An algorithm for stabilization of fractional-order time delay systems using fractional-order PID controllers," IEEE Transactions on Automatic Control, vol. 52, no. 10, pp. 1964-969, 2007. doi:10.1109/tac.2007.906243.

[16] Shiqi Zheng, Xiaoqi Tang, Bao Song, "A graphical tuning method of fractional order proportional integral derivative controllers for interval fractional order plant," Journal of process control, vol. 24, 2014. doi:10.1016/j.jprocont.2014.08.011.

[17] Y.-C. Cheng and C. Hwang, "Stabilization of unstable first-order timedelay systems using fractional-order PD controllers," J. Chinese Inst, vol. 29, no. 2, pp. 241-249, 2006. doi: 10.1080/02533839.2006.9671121.

[18] Zhong, Jianpeng, and Lichuan Li, "Tuning fractional-order controllers for a solid-core magnetic bearing system," IEEE Transactions on Control Systems Technology, vol. 23, no. 4, pp, 2015. 1648-1656., doi:10.1109/tcst.2014.2382642.

[19] Zhong, Jianpeng, and Lichuan Li, "Fractional-order system identification and proportional-derivative control of a solid-core magnetic bearing," ISA Transactions, vol. 53, no. 4, 1232-242, 2014. doi:10.1016/j.isatra.2014.05.008.

[20] Chen, Seng-Chi, Van-Sum Nguyen, Dinh-Kha Le, and Nguyen Thi Hoai Nam, "Nonlinear control of an active magnetic bearing system achieved using a fuzzy control with radial basis function neural network," Journal of Applied Mathematics, pp. 1-18, 2014. doi:10.1155/2014/272391.

[21] Le, Anh, Veerajagadheswar Prabakaran, Vinu Sivanantham, and Rajesh Mohan, "Modified a-star algorithm for efficient coverage path planning in tetris inspired self-reconfigurable robot with integrated laser sensor," Sensors, vol. 18 , no. 8, 2018. doi:10.3390/s18082585.

[22] Rashid, Tarique, Sunil Kumar, Akshay Verma, Prateek Raj Gautam, and Arvind Kumar, "Pm-EEMRP: Postural movement based energy efficient multi-hop routing protocol for intra wireless body sensor network (IntraWBSN)." TELKOMNIKA, vol. 16, no. 1, pp. 166, 2018. doi:10.12928/telkomnika.v16i1.7318.

[23] A. F. Morabito, "Power synthesis of mask-constrained shaped beams through maximally-sparse planar arrays," Telkomnika (Telecommunication Computing Electronics and Control), vol. 14, no. 4, pp. 1217-1219, 2016.

[24] Phu Tran Tin, Tran Hoang Quang Minh, Tran Thanh Trang, Nguyen Quang Dung, "Using real interpolation method for adaptive identification of nonlinear inverted pendulum system," International Journal of Electrical and Computer Engineering (IJECE), vol. 9, no. 2, pp. 1078-1089, 2019. DOI: 10.11591/ijece.v9i2.pp.1078-1089.

[25] Phu Tran Tin, Le Anh Vu, Minh Tran, Nguyen Quang Dung, Tran Thanh Trang, "Optimal tuning PID controller of unstable fractional order system by desired transient characteristics using RIM," International Journal of Electrical and Computer Engineering, vol. 14, no. 3, pp. 1177-1188, 2019. http://doi.org/10.11591/ijeecs.v14.i3.pp11771188 . 